\title{
Cirugía transoral robótica en síndrome de apnea e hipoapnea obstructiva del sueño
}

\author{
Transoral robotic surgery in obstructive sleep apnea/hypopnea syndrome
}

\author{
Sebastián Castro M. ${ }^{1}$, Dafne Segall K. ${ }^{1}$, Felipe Cardemil M. ${ }^{1,2}$
}

\begin{abstract}
Resumen
El síndrome de apnea e hipoapnea obstructiva del sueño (SAHOS) es una condición frecuente, que se asocia a importantes consecuencias negativas. El tratamiento inicial suele ser médico, pero en pacientes seleccionados la cirugía tiene excelentes resultados. En los últimos años se ha desarrollado la cirugía robótica transoral para tratar a pacientes con obstrucción anatómicas a nivel retrolingual, hipofaríngeo o supraglótico, mediante amigdalectomía lingual, reducción de base de lengua y/o supraglotoplastía. En pacientes seleccionados, esta técnica ha mostrado iguales o mejores resultados funcionales que las técnicas clásicas, junto con una baja tasa de complicaciones posoperatorias, sin embargo, implica un mayor costo asociado. A pesar de esto último, es una técnica válida y prometedora en el campo del SAHOS.

Palabras clave: SAHOS, cirugía, transoral, robótica.
\end{abstract}

\begin{abstract}
Obstructive sleep apnea and hypopnea syndrome (OSAHS) is a common condition that is associated with many negative consequences. First line treatment is usually medical management, but surgery is indicated for selected patients with retrolingual, hipopharyngeal or supraglottic obstruction. Transoral robotic surgery has been developed in the last years for the surgical treatment, through tongue base reduction and/ or supraglottoplasty. In selected patients, this technique has shown equal or better functional outcomes than conventional techniques, along with a low rate of postoperative complications, however it implies a higher associated cost. Despite the latter, it is a valid and promising technique in the field of OSAHS.
\end{abstract}

Keywords: OSAHS, TORS, transoral, robotic, surgery.

\author{
'Servicio de Otorrinolaringología, \\ Hospital San Juan de \\ Dios, Departamento de \\ Otorrinolaringología, Facultad de \\ Medicina, Universidad de Chile. \\ Santiago, Chile. \\ ${ }^{2}$ Departamento de \\ Otorrinolaringología, Clínica Las \\ Condes. Santiago, Chile. \\ Los autores declaran no tener \\ conflictos de interés. \\ Recibido el 20 de octubre de 2020. \\ Aceptado el 1 de diciembre de \\ 2020. \\ Correspondencia: \\ Felipe Cardemil M. \\ Departamento de \\ Otorrinolaringología \\ Departamento de Oncología Básico- \\ Clínica \\ Facultad de Medicina, Universidad \\ de Chile \\ Santiago, Chile. \\ Email: felipecardemil@med.uchile.cl
}

\section{Introducción}

El síndrome de apnea e hipoapnea obstructiva del sueño (SAHOS) es una condición caracterizada por la disminución del flujo aéreo secundario a una obstrucción temporal, parcial o total, de la vía aérea superior ${ }^{1}$. La prevalencia de esta patología es variable, encontrándose entre un $9 \%$ a un $38 \%$ en la población general, tomando como corte un índice de apnea/hipoapnea (IAH) $\geq$ a 5 , siendo mayor en el sexo masculino, en personas de edad avanzada y en pacientes obesos ${ }^{1,2}$.

Se ha planteado que el SAHOS representa un problema de salud pública, debido a que se ha asociado con una mayor morbimortalidad producto de alteraciones en tres esferas: por un lado, mayor riesgo cardiovascular, definido por mayor riesgo de infarto agudo al miocardio, hipertensión arterial y accidente cerebrovascular. Por otro lado, mayor riesgo metabólico, caracterizado por mayor riesgo de resistencia a la insulina y dificultad de control del peso. Por último, un mayor riesgo de accidentalidad en vehículos motorizados y maquinarias, mayor accidentalidad laboral, menor rendimiento escolar y laboral, además de alteraciones de la esfera sexual, somnolencia diurna, irritabili- 
dad, y algunas asociaciones con trastornos del ánimo, como depresión, entre otros ${ }^{3,4}$. Todo esto hace que el manejo oportuno de esta enfermedad sea fundamental para disminuir consecuencias y costos asociados ${ }^{1}$.

Dentro de los pilares de tratamiento se encuentra el manejo médico, el cual incorpora medidas de cambios de estilo de vida, higiene de sueño y utilización de dispositivos que proveen ventilación con presión positiva (PAP). Los dispositivos PAP pueden ser de presión continua o CPAP; presión continua bi-nivel o BPAP, con una menor presión positiva espiratoria que inspiratoria; o presiones auto ajustables según eventos obstructivos detectados, donde encontramos APAP y auto-BPAP ${ }^{4}$. Esto ha demostrado ser efectivo para lograr la normalización del sueño, considerándose tradicionalmente como un tratamiento adecuado en la mayoría de los pacientes, especialmente en aquellos con somnolencia excesiva o con SAHOS moderado a severo ${ }^{4,5}$.

El efecto de los dispositivos PAP en la reducción de eventos cardiovasculares sigue siendo un tema controversial, debido a resultados contradictorios entre diferentes estudios ${ }^{4}$. El ensayo clínico aleatorizado (ECA) más grande a la fecha fue el publicado el año 2016 por McEvoy y cols., donde se observó que la utilización de CPAP en pacientes con SAHOS moderado a severo no redujo el riesgo de eventos cardiovasculares en pacientes con enfermedad cardiovascular establecida, con un seguimiento promedio de 3,7 años ${ }^{6}$. Además, un metaanálisis reciente realizado por Patil y cols. que evaluó ECAs que abordaban esta pregunta, sugirió que no había reducción de los eventos cardiovasculares con el uso de los dispositivos PAP en pacientes con SAHOS. No obstante, los resultados de un metaanálisis de ensayos clínicos no aleatorizados sugirieron una reducción en los eventos cardiovasculares en pacientes con SAHOS con el uso de $\mathrm{PAP}^{4}$. Por esta discrepancia en los resultados de la literatura, se considera que aún se necesitan más estudios para determinar la influencia real del tratamiento con PAP sobre el riesgo cardiovascular. Sin embargo, el estándar de tratamiento en el mundo sigue siendo el uso de dispositivos PAP para el manejo de pacientes con SAHOS, especialmente los casos severos y moderados, pero aún hace falta más evidencia que respalde la efectividad de este tratamiento en la reducción de mortalidad por eventos cardiovasculares.

A pesar de que el manejo con dispositivos PAP es de primera línea en una gran proporción de pacientes, en algunos casos seleccionados el tratamiento quirúrgico es una opción favorable ${ }^{5}$. Los motivos por los cuales la cirugía puede ser una opción válida son por falta de adherencia al dispositivo PAP, para permeabilización de la vía aérea, para adaptación de dispositivos PAP o para cirugía de tratamiento definitivo a nivel del complejo nasal-orofaríngeo y, en algunos casos, para abordar obstrucciones a nivel retrolingual, hipofaríngeo y/o laríngeo. Para detectar aquellos pacientes candidatos a este tipo de tratamiento, la evaluación debe ser clínica, radiológica y funcional endoscópica, tanto en estado de vigilia como mediante sueño espontáneo o inducido, con el fin de lograr encontrar el sitio de obstrucción anatómica asociado ${ }^{1,5}$.

Dentro del tratamiento quirúrgico de la base de lengua, hipofaringe y supraglotis existen distintas modalidades de manejo transoral para abordar diferentes sitios de obstrucción anatómica, tales como técnica fría, microcirugía transoral láser, ablación con radiofrecuencia o coblator y cirugía transoral robótica (TORS). El objetivo del presente artículo es describir la utilidad y resultados de TORS en SAHOS con el fin de señalar el estado de arte de esta técnica quirúrgica.

\section{Historia de la cirugía robótica}

La cirugía robótica ha tenido una evolución importante en las últimas décadas desde su primera introducción en 1985 con el robot PUMA 560 , un brazo robótico con el cual se realizaron procedimientos neuroquirúrgicos guiados por tomografía computarizada. Esta evolución ha permitido el desarrollo de nuevas tecnologías con ventajas cualitativas con respecto a otras técnicas quirúrgicas, logrando incluso incorporar sistemas telerrobóticos que permiten cirugías a distancia desde el año $2001^{7}$.

Actualmente, el sistema robótico más comúnmente comercializado es el sistema robótico daVinci, el cual ha dominado el mercado robótico quirúrgico en la última década, con 
más de 5.000 unidades vendidas. Este sistema fue primeramente utilizado en cardiocirugía para la realización de cirugía de derivación aortocoronaria cerrada. No obstante, su aplicación se ha extendido ampliamente, llevando a su utilización por varias especialidades quirúrgicas incluyendo urología, ginecología, traumatología y ortopedia, cirugía torácica, cirugía general, cardiocirugía y cirugía de cabeza y cuello ${ }^{7,8}$.

Este sistema fue introducido al campo de la cirugía de cabeza y cuello el año 2003, cuando Haus y cols. utilizaron este robot en modelos animales de cerdos con el fin de realizar parotidectomías, submandibulectomías, disecciones cervicales y timectomías ${ }^{8}$. Pero no fue hasta el 2005 donde se utilizó por primera vez en un paciente vivo para la remoción de un quiste vallecular por Weinstein y cols. en la Universidad de Pensilvania. Desde entonces, se han publicado varios estudios y artículos que dan cuenta de la eficacia y seguridad de su uso en cirugías de orofaringe, hipofaringe, laringe y otros (espacio parafaríngeo, espacio retrofaríngeo, base de cráneo y abordajes de cuello robóticos), por lo que su rol en dichas áreas está siendo reevaluado constantemente ${ }^{8}$.

La cirugía robótica en cabeza y cuello tiene varias ventajas sobre otras técnicas quirúrgicas, donde se destaca la magnificación de las imágenes hasta 10-12 veces, lo que permite evaluar y detectar lesiones subcentimétricas; visión en 3 dimensiones (3D); manipulación tisular precisa y posibilidad de resección en bloc; movimientos con 7 grados de libertad; filtro de movimiento con ausencia de temblor en los brazos quirúrgicos; ajuste de movimientos; además de la posibilidad de realizar telecirugías ${ }^{7}$.

Es por esta razón que la cirugía robótica ha tenido una expansión importante en la última década en esta área. La TORS está aprobada desde el 2009 por la U.S. Food and Drug Administration para el enfrentamiento de patologías benignas y malignas clasificadas como T1-T2 en orofaringe. Sin embargo, existen varios estudios retrospectivos y series de casos que avalan su utilización en patologías benignas y malignas seleccionadas en laringe e hipofaringe, específicamente en metástasis cervical con primario desconocido y frente a neoplasias malignas supraglóticas candidatas a laringectomía supraglótica ${ }^{8}$. Por este motivo, el campo de la TORS se encuentra en expansión continua ${ }^{8}$.

En el área de condiciones benignas, la TORS se ha utilizado para el manejo de pacientes seleccionados con SAHOS con el fin de abordar compromisos obstructivos anatómicos multinivel, especialmente en base de lengua y supraglotis, por razones que comentaremos a continuación.

\section{Cirugía robótica en SAHOS}

Uno de los problemas del uso de CPAP e incluso de APAP es la adherencia a este tratamiento, la cual varía entre $50 \%$ a $70 \%$. Es por esa razón que se han buscado tratamientos alternativos para aquellos pacientes que no toleran el dispositivo, dentro de los cuales existen los tratamientos quirúrgicos. El tipo de cirugía que se realice varía dependiendo tanto del nivel, de la severidad, como del tipo de obstrucción de la vía aérea superior, lo que se detecta mediante análisis endoscópico tanto en vigilia como en sueño espontáneo o inducido ${ }^{1,9}$.

En el caso de la obstrucción de la vía aérea superior a nivel retrolingual, especialmente dependiente de base de lengua, existen múltiples técnicas quirúrgicas para abordar este problema. Éstas se pueden dividir en 2 grupos: a) Procedimientos tipo debulking, como la glosectomía en línea media, la ablación por radiofrecuencia o coblator y la amigdalectomía lingual mediante TORS y b) Procedimientos de reposicionamiento de tejidos, como suspensión lingual, avance del geniogloso y avance maxilomandibular ${ }^{6}$. Además de éstos, últimamente ha ganado popularidad el uso de sistemas de estimulación del nervio hipogloso ${ }^{10}$.

La introducción de TORS en SAHOS fue primeramente reportada en el año 2010 cuando Vicini y cols. publicaron los resultados preliminares obtenidos tras realizar TORS para abordar base de lengua en 10 pacientes con SAHOS. Obtuvieron buenos resultados objetivos, disminuyendo el promedio de IAH desde 38,3 a 20,6. Además, obtuvieron buenos resultados funcionales, en cuanto a dolor posoperatorio, deglución y calidad de vida, con un puntaje de Epworth Sleepiness Scale (ESS) preoperatorio y 
posoperatorio de 12,4 y 6,9 respectivamente, además de baja tasa de complicaciones ${ }^{11}$. Similares resultados se han reportado en otros estudios, dando cuenta de una mejoría en los parámetros antes mencionados en pacientes seleccionados ${ }^{12,13}$.

La selección de los pacientes para TORS es esencial para lograr buenos resultados. Es por esta razón que es de suma importancia evaluar preoperatoriamente el grado de hipertrofia de base de lengua e idealmente determinar el volumen de las amígdalas linguales, con el fin de estimar la cantidad de tejido resecable sin dañar el músculo subyacente ${ }^{14}$. Dado lo anterior, los mejores candidatos, especialmente para cirujanos principiantes, son aquellos con hipertrofia de base de lengua baja (en el área de la epiglotis-vallécula), tipo linfoide y localizada, regla llamada Triple L (low, lymphoid, localized $)^{14}$.

Existen algunas condiciones que contraindican la reducción de base de lengua con TORS. Dentro de las contraindicaciones absolutas, se destaca principalmente a aquellos pacientes que tengan un buen control de su enfermedad con manejo médico, o aquellos pacientes que presenten comorbilidades cardiovasculares inestables, con un puntaje American Society of Anesthesiologists (ASA) $>2$, o que presenten enfermedades neuromusculares progresivas, entre otras. Por otro lado, en cuanto a sus contraindicaciones relativas, se encuentran condiciones locales, principalmente pacientes con baja exposición transoral específicamente secundario a micrognatia o macroglosia. Sin embargo, existen centros donde no excluyen a estos grupos de pacientes ${ }^{14}$.

\section{Técnica quirúrgica TORS}

Antes de abordar la técnica quirúrgica de TORS, es importante señalar breves aspectos de la anatomía de base de lengua y su clasificación actual.

\section{Anatomía quirúrgica de base de lengua}

Aun cuando un análisis detallado de la anatomía se escapa del objetivo de la presente revisión, es importante tener en cuenta que el cirujano debe estar entrenado para visualizar la anatomía desde "dentro hacia afuera" (insi- de-out), con el fin de evitar daños a estructuras neurovasculares importantes, teniendo en cuenta que la mayoría de los cirujanos están más familiarizados entendiendo la anatomía desde lateral a medial ${ }^{15}$.

La base de lengua limita anteriormente con la lengua oral, separado por el sulcus terminalis que tiene forma de $\mathrm{V}$ invertida, donde se relacionan con las papilas circunvaladas. En el ápex de este sulcus se encuentra el foramen cecum. Esta base de lengua se une tanto al hioides como a la mandíbula mediante el músculo hiogloso, geniogloso y la membrana hioglosal que está en continuidad con la epiglotis por el ligamento glosoepiglótico ${ }^{16}$.

La superficie de la base de lengua se compone de una membrana mucosa linfoide, determinada por las amígdalas linguales, y por una parte muscular, dada principalmente por la musculatura intrínseca y extrínseca lingual y por la inserción del músculo estilogloso a la musculatura intrínseca en su región posterolateral ${ }^{15,16}$.

En cuanto a las estructuras vasculares, el principal elemento es la arteria lingual, la cual se origina desde la arteria carótida externa, transcurre lateral al cuerno mayor del hioides, lateral al músculo constrictor medio de la faringe, pasando debajo y lateral al músculo estilogloso dirigiéndose, finalmente, en dirección anteromedial, para dividirse en sus ramas dorsales linguales, que van mediales al músculo estilogloso, y profundas en la lengua ${ }^{15}$.

Finalmente, en relación con las estructuras nerviosas, es importante señalar que tanto el nervio hipogloso como el glosofaríngeo son las principales estructuras que pueden ser dañadas en este tipo de cirugías. El primero corre lateral al curso de la arteria lingual y al músculo hiogloso, en la región posterolateral de la base de lengua. Mientras que el glosofaríngeo, se encuentra posterior y lateral al músculo estilogloso en la unión de la base de lengua, entre este músculo y el ligamento estilohioideo, y luego se divide en ramas dirigidas hacia la base de lengua y hacia la pared lateral faríngea ${ }^{15,17}$.

\section{Clasificación de amígdalas linguales}

Para evaluar las amígdalas linguales se debe examinar al paciente con la lengua en distintas posiciones, aunque generalmente 
éstas son mejor visualizadas con la lengua en protrusión. La clasificación por grados de las amígdalas linguales, según Friedman, va de 0 a 4 y depende de su relación con la base de la lengua, como se describe en la Tabla $1^{18}$.

\section{Reducción de base de lengua}

Aunque existen variaciones dependiendo del centro en donde se realice, se describirá la técnica más aceptada actualmente. Esta cirugía se realiza bajo anestesia general, estableciendo de forma primaria una vía aérea segura. En casos excepcionales, si se considera necesario, se puede considerar realizar una traqueostomía preoperatoria de seguridad, pero generalmente se realiza intubación oro o nasotraqueal. Con el fin de disminuir el edema en vía aérea superior y vómitos posoperatorios se recomienda la utilización de corticoides endovenosos ${ }^{14}$.

Es importante considerar los instrumentales utilizados en este tipo de cirugía, donde se encuentra una óptica de $12 \mathrm{~mm}$ de 30 grados, electrocauterio monopolar para disecar y coagular, un disector tipo Maryland para tomar y disecar, además de un sistema de aspiración doble. Se recomienda usar un bajo aumento y una visión angular amplia, para permitir la visión en 3 dimensiones de la anatomía ${ }^{12,19}$. Además, es clave el uso de dispositivos abreboca adecuados, siendo los más utilizados el retractor-abreboca Feyh-Kastenbauer modificado por Weinstein y O'Malley (FK-WO) u otros, como el abreboca Davis Meyer.

El paciente debe ser acomodado en la llamada "posición de olfateo", con una flexión de cuello y extensión de cabeza. Una vez posicionado tanto el equipo quirúrgico como el paciente, se comienza el procedimiento fijando la punta de la lengua con sutura de seda 0 junto con la instalación del abrebocas bajo luz frontal, con el fin de traicionar el cuerpo de la lengua ${ }^{10}$. Una vez posicionada la óptica sobre la base de lengua, se divide en línea media la base de lengua entre foramen cecum y ligamento glosoepiglótico. Al tomar como punto de referencia el foramen cecum, se intentan preservar las papilas circunvaladas y con ellos maximizar la sensación de gusto en el posoperatorio ${ }^{12,19}$.

Posteriormente, se reduce tejido linfoide bilateralmente por medio de una amigdalectomía lingual completa (similar a lo que sería
Tabla 1. Clasificación de amígdalas linguales según Friedman et al. ${ }^{18}$

Grado 0 Ausencia de tejido linfoide en la base de la lengua

Grado 1 Tejido linfoide disperso en la base de la lengua

Grado 2 Tejido linfoide que cubre toda la base de la lengua, pero con espesor vertical limitado

Grado 3 Tejido linfoide significativamente elevado que cubre la totalidad de la base de la lengua, aproximadamente de 5 a $10 \mathrm{~mm}$ de espesor

Grado 4 Tejido linfoide que cubre la totalidad de la lengua, de $1 \mathrm{~cm}$ o más de grosor, que se eleva por encima de la punta de la epiglotis

una amigdalectomía palatina, pero sobre las amígdalas localizadas en la base de la lengua), lo que se puede asociar o no a resección de tejido muscular de base lingual, dependiendo del compromiso de éste. En caso de disección de músculo, ésta puede ser de hasta $10 \mathrm{~mm}$ de profundidad, sin causar secuelas. Primero se identifican los bordes superior, lateral e inferior de la tonsila lingual derecha y se marcan con electrocauterio. Se realiza la amigdalectomía lingual derecha "en bloc", de superior a inferior. Luego se invierten los brazos del robot y herramientas y se realiza el mismo procedimiento en la amígdala lingual izquierda. Finalmente, se debe realizar una inspección del campo en búsqueda de obstrucción residual ${ }^{12,19}$.

Si la visión de la glotis no es completa, con una visualización tipo Cormack y Lehane $\geq$ grado II, se realiza glosectomía de línea media, de hasta $5 \mathrm{~mm}$ de ancho por $5 \mathrm{~mm}$ de profundidad adicionales, terminando en forma de $\mathrm{T}^{19,20}$. El objetivo de la cirugía es lograr un Cormack y Lehane grado I o II y/u obtener al menos $7 \mathrm{ml}$ de resección lingual, teniendo en cuenta que en promedio se resecan $10 \mathrm{ml}^{14}$.

\section{Supraglotoplastía}

En aquellos pacientes que presenten una retropulsión de la epiglotis que colapse la entrada laríngea o en el caso de tejido redundante supraglótico, se puede plantear una supraglotoplastía. Esta técnica, se realiza en el mismo acto quirúrgico, toma aproximadamente 15 minutos adicionales a la reducción de base de lengua ${ }^{14}$. Se realiza una división vertical de la 


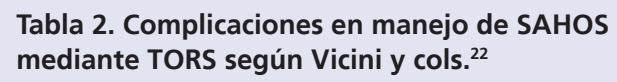

Alteraciones gustativas transitorias

Sangrado posoperatorio

$14,2 \%$ $5 \%$
Sangrado tardío

Estenosis faríngea

$2,9 \%$

$0,4 \%$

Complicaciones extraídas desde estudio de Vicini y cols. de 243 pacientes tratados mediante TORS 22 epiglotis suprahioídea en línea media, desde la punta hasta $5 \mathrm{~mm}$ sobre el nivel de la base vallecular. Luego, se realiza un corte horizontal hacia lateral sobre los pliegues aritenoepiglóticos para minimizar el riesgo de sangrado y el riesgo de aspiración ${ }^{19}$. La cicatrización se logra bajo segunda intención lo que puede conllevar el desplazamiento anterior epiglótico, lo que puede aumentar el riesgo de aspiración si se reseca de forma excesiva ${ }^{20}$.

\section{Manejo posoperatorio}

Los pacientes sometidos a esta técnica quirúrgica debieran idealmente permanecer hospitalizados en Unidad de Cuidados Intermedios (UCI) o en unidades stepdown con vigilancia estricta por enfermería por al menos 24 horas. Esto debido a que el paciente se debe monitorizar por riesgo de sangrado y compromiso de vía aérea tardío, que se presenta en las primeras 24 horas. Posteriormente se pueden trasladar a una sala básica para seguir en observación por 24 horas adicionales. Como en todo procedimiento quirúrgico, se debe proveer un manejo adecuado del dolor, por 3 a 5 días. Se inicia alimentación al segundo día posoperatorio, con régimen líquido, y sólido a partir del séptimo día ${ }^{19,21}$.

\section{Complicaciones}

Con relación a las complicaciones relacionadas, una de las series más largas fue la publicada por Vicini y cols., donde evaluaron 243 pacientes que se sometieron a TORS por
SAHOS entre el 2008 y 2012 en siete instituciones. Las complicaciones más frecuentes en este estudio fueron las alteraciones gustativas transitorias reportándose en un 14,2\% de los casos, todos se recuperaron antes del año. Por otro lado, el sangrado posoperatorio se reportó en un $5 \%$, junto con sangrado tardío en un $2,9 \%$, estos últimos fueron autolimitados y no requirieron intervención. Sólo $1,7 \%$ de los pacientes requirieron una nueva intervención quirúrgica para controlar el sangrado. Finalmente, como complicación tardía se observó cicatriz y estenosis faríngea en $0,4 \%$ de los procedimientos, esta última que requiere un manejo complejo para su reparación (Tabla 2$)^{22}$.

Otra complicación menos frecuente es el daño neural inadvertido, el cual se ha reportado principalmente en el manejo de neoplasias orofaríngeas con TORS. En ese sentido se ha reportado daño del nervio lingual transitorio en un $0,6 \%$ de los casos, además de daño del nervio hipogloso en $0,9 \%$ de los casos de manera transitoria y en un $0,1 \%$ de los casos de forma permanente, lo que se explica tanto por la técnica quirúrgica utilizada, como también por la compresión que ejerce el abrebocas sobre la superficie lingual ${ }^{23}$.

\section{Éxito de TORS en SAHOS}

Como fue señalado anteriormente, en pacientes seleccionados, la TORS presenta una buena efectividad, donde el éxito reportado en la literatura varía entre un $45 \%$ a $67 \%$ en pacientes con SAHOS severo con obstrucción retrolingual o supraglótica. Friedman y cols. compararon los resultados de Z-palatoplastía asociado a TORS, a reducción de base de lengua con radiofrecuencia y a resección con coblator. Se observaron mejores resultados, estadísticamente significativos, en cuanto a disminución de IAH posoperatorio y mejoría en saturación de oxígeno con Z-palatoplastía + TORS, pero la recuperación de la deglución, retorno a actividades, días de hospitalización y costos de la cirugía asociados a TORS fueron mayores ${ }^{24}$.

Más recientemente, Miller y cols. realizaron una revisión sistemática y metaanálisis con el objetivo de evaluar la efectividad de TORS en reducción de base de lengua. Un 
total de seis artículos con 353 pacientes cumplieron los criterios de inclusión. Cabe señalar que en estos estudios se incorporaron pacientes que se sometían a reducción de base de lengua por TORS asociándose o no a otra cirugía, donde sólo 57 pacientes se sometieron solamente a este procedimiento. Analizando los datos de estos 353 pacientes se observó una mejora significativa posquirúrgica en comparación con el basal, para IAH, desde un promedio de 44,3 vs 17,8 posquirúrgico; para el puntaje de ESS, con un basal promedio de 12,9 vs. 5,8 puntos posoperatorio; para la saturación de oxígeno menor registrada, con un basal de hasta un promedio de $79,0 \%$ vs. $84,1 \%$ posoperatorio; y para escala visual análoga para ronquido. Por otro lado, la tasa de éxito quirúrgico, definido como una reducción sobre el $50 \%$ del IAH con un IAH posoperatorio $<20$, fue $68,4 \%{ }^{9}$.

Interesantemente, se han asociado factores predictores de peor éxito quirúrgico en TORS. Lin y cols., publicaron un estudio retrospectivo analizando 39 pacientes que se sometieron a reducción de base de lengua vía TORS con o sin epiglotectomía parcial con o sin uvulopalatofaringoplastía. Ellos encontraron una tasa de éxito quirúrgico, definida como una reducción sobre el 50\% del IAH con un IAH posoperatorio $<20$ con resolución de la somnolencia diurna, en $53,8 \%$ de los pacientes. Sin embargo, si se estratifican por índice de masa corporal (IMC), aquellos que tenían un IMC $<30$ tenían una excelente tasa de éxito quirúrgico en un $88,2 \%$, mientras que aquellos con un IMC $>40$ tenían pobres resultados son sólo $16,7 \%$ de tasa de éxito quirúrgico. Por otro lado, pacientes con un IAH $<60$ tenían una tasa de respuesta de $67,9 \%$, mientras que aquellos con un IAH $>60$ tenían una tasa de sólo $18,2 \%$. Finalmente, aquellos que no tenían colapso lateral velofaríngeo tenían mayor una tasa de éxito quirúrgico $(66,74 \%)$ comparado con aquellos que tenían dicho colapso, con una tasa de $25,0 \%{ }^{25}$.

A pesar de los resultados señalados anteriormente, hay que considerar que estos son derivados desde estudios retrospectivos, los cuales tienen riesgo de sesgos que pueden influir en los resultados finales. Por lo que se necesitan ECAs para demostrar la eficacia real de la cirugía multinivel, y específicamente de
TORS en el manejo de pacientes con SAHOS. Actualmente se está cursando uno de los primeros ECA en cirugía multinivel en pacientes con SAHOS que presentan una mala respuesta a CPAP, este estudio denominado SAMS trial, buscará responder la pregunta si una cirugía multinivel abordando tanto la obstrucción retropalatina, mediante una uvulopalatofaringoplastía modificada, como la obstrucción retrolingual, mediante radiofrecuencia, es mejor que el manejo médico del SAHOS, con un mejor nivel de evidencia; además este estudio pretende evaluar la costo-efectividad del manejo quirúrgico multinivel en comparación con el manejo médico ${ }^{26}$.

\section{Recurrencia o persistencia}

Aquellos pacientes que persisten o recurren en cuanto a sintomatología, se deben someter a un nuevo estudio endoscópico (idealmente mediante drug-induced sedated endoscopy, DISE) para determinar el nivel de obstrucción, asociándose o no a exámenes complementarios como tomografía computarizada o resonancia magnética con protocolos estandarizados para SAHOS. En estos pacientes, se ha visto que una obstrucción retropalatina es más común que la retrolingual en una proporción de 2:144.

En el caso particular del compromiso retrolingual, se han descrito 2 tipos de patrones de obstrucción más frecuentes, visualizados mediante DISE: a) Tipo base de lengua superior o colapso de lengua oral, donde la lengua contacta paladar blando generando un colapso palatino secundario; b) Tipo inestabilidad lingual lateral que provoca un colapso en línea media de la base de lengua. Estos patrones se pueden abordar nuevamente de forma quirúrgica o, en su defecto, con tratamiento médico ${ }^{14}$.

\section{Costos}

Actualmente el costo del sistema robótico daVinci es menor. Inicialmente, según cifras estimadas en 2009, el costo de este sistema junto con la actualización de su software era de US 1,5 millones de dólares, junto con US 150.000 dólares anuales por mantención ${ }^{27}$. Sin embargo, los costos se han abaratado con el paso del tiempo, sumado a que el sistema daVinci está más disponible para otras especialidades o incluso en otorrinolaringología 
para enfermedades oncológicas, por lo que la disponibilidad ha aumentado, así como el entrenamiento de los equipos humanos, y los brazos robóticos pueden reutilizarse hasta 20 veces cada uno, con un costo estimado de US 500 dólares por el equipamiento desechable ${ }^{29}$.

Los estudios que evalúan el costo-efectividad de utilizar TORS en SAHOS, cáncer orofaríngeo o cavidad oral de etapa temprana son controvertidos. Algunos estudios que evalúan los costos asociados a TORS en este último grupo, muestran resultados contradictorios ${ }^{27}$. No obstante, se observa que la inversión inicial para la adquisición del sistema robótico, junto con el entrenamiento del personal, es uno de los principales factores que hacen que TORS sea una modalidad de mayor costo que otros procedimientos quirúrgicos. A pesar de la existencia de evidencia que indica que puede disminuir la estadía hospitalaria por 1 día en promedio, esto no es suficiente para señalar que disminuiría los costos, además de estar basado en evidencia de baja calidad y no estar asociado con un análisis de costo-efecti$\operatorname{vidad}^{27,28}$. Por este motivo, consideramos que los datos de costo-efectividad y costo-beneficio asociado al uso de TORS como herramienta estándar en el manejo de pacientes con SAHOS debe ser analizada como parte del proceso de riesgo-beneficio caso a caso ${ }^{29}$.

\section{Conclusión}

El SAHOS es una condición prevalente donde el tratamiento de primera línea en la mayoría de los casos es médico. Sin embargo, la adherencia al tratamiento con dispositivos PAP no es óptima. Por esta razón el tratamiento quirúrgico emerge como una opción válida en un grupo seleccionado de pacientes. La TORS es una técnica validada para el abordaje de obstrucción retrolingual en el manejo de la vía aérea multinivel, con complicaciones infrecuentes, menores tiempos operatorios asociados y tasas de éxitos adecuadas en pacientes seleccionados. Consideramos que TORS es una técnica prometedora por las ventajas cualitativas asociadas, por lo que se debe extender su enseñanza y utilización en este campo con el fin de ofrecer los mejores resultados hacia nuestros pacientes.

\section{Bibliografía}

1. Huon L, Guilleminault C. Chapter 2: Signs and Symptoms of Obstructive Sleep Apnea and Upper Airway Resistance Syndrome. En: Friedman P. Sleep Apnea and Snoring: Surgical and Non Surgical Therapy. Filadelfia: Ed. Elsevier, 2020; 6-12.

2. Senaratna CV, Perret JL, Lodge CJ y cols. Prevalence of obstructive sleep apnea in the general population: A systematic review. Sleep Med Rev. 2017;34:70-81. doi:10.1016/j.smrv.2016.07.002.

3. Welman A, Redline S. Chapter 319: Sleep Apnea. In: Kasper y cols. Harrison: Principles of Internal Medicine, 19th Edition. Ed. McGraw Hill, 2015; Volume 2: p 1723-1727.

4. Patil SP, Ayappa IA, Caples SM, Kimoff RJ, Patel SR, Harrod CG. Treatment of Adult Obstructive Sleep Apnea With Positive Airway Pressure: An American Academy of Sleep Medicine Systematic Review, Meta-Analysis, and GRADE Assessment. J Clin Sleep Med. 2019;15(2):301-334. doi: 10.5664/ jcsm.7638.

5. Sarber K, Lam D, Ishman S. Chapter 15: Sleep Apnea and Sleep Disorders. In: Flint, Paul W., Haughey, Bruce H., eds. Cummings Otolaryngology. Philadelphia: Elsevier, 2020; 215-235.

6. McEvoy RD, Antic NA, Heeley E, Luo Y, Ou Q, Zhang X y cols. CPAP for Prevention of Cardiovascular Events in Obstructive Sleep Apnea. N Engl J Med. 2016;375(10):919-31. doi: 10.1056/ NEJMoa1606599.

7. Garas G, Arora A. Robotic Head and Neck Surgery: History, Technical Evolution and the Future. ORL J Otorhinolaryngol Relat Spec. 2018;80(3-4):117-124. doi:10.1159/000489464.

8. Bekeny JR, Ozer E. Transoral robotic surgery frontiers. World J Otorhinolaryngol Head Neck Surg. 2016;2(2):130-135. doi:10.1016/j.wjorl.2016.05.001.

9. Miller SC, Nguyen SA, Ong AA, Gillespie MB. Transoral robotic base of tongue reduction for obstructive sleep apnea: A systematic review and meta-analysis. Laryngoscope. 2017;127(1):258-265. doi:10.1002/lary.26060.

10. Kompelli AR, Ni JS, Nguyen SA, Lentsch EJ, Neskey DM, Meyer TA. The outcomes of hypoglossal nerve stimulation in the management of OSA: A systematic review and meta-analysis. World J Otorhinolaryngol Head Neck Surg. 2018;5(1):41-48. doi:10.1016/j. wjorl.2018.04.006.

11. Vicini C, Dallan I, Canzi P y cols. Transoral robotic tongue base resection in obstructive sleep apnoeahypopnoea syndrome: a preliminary report. $O R L$ 
J Otorhinolaryngol Relat Spec. 2010;72(1):22-7. doi: $10.1159 / 000284352$.

12. Vicini C, Dallan I, Canzi P y cols. Transoral robotic surgery of the tongue base in obstructive sleep ApneaHypopnea syndrome: anatomic considerations and clinical experience. Head Neck. 2012;34(1):15-22. doi: 10.1002/hed.21691.

13. Lin HS, Rowley JA, Badr MS, y cols. Transoral robotic surgery for treatment of obstructive sleep apneahypopnea syndrome. Laryngoscope. 2013;123(7):18111816. doi:10.1002/lary.23913.

14. Vicini C, Montevecchi F, Meccariello G. Chapter 55: Transoral Robotic Surgery (TORS) for OSA. En: Friedman P. Sleep Apnea and Snoring: Surgical and Non Surgical Therapy. Filadelfia: Ed. Elsevier, 2020; 315-318.

15. Gun R, Durmus K, Kucur C, Carrau RL, Ozer E. Transoral Surgical Anatomy and Clinical Considerations of Lateral Oropharyngeal Wall, Parapharyngeal Space, and Tongue Base. Otolaryngol Head Neck Surg. 2016;154(3):480-485. doi:10.1177/0194599815625911

16. Friedman M, Kelley K, Maley A. Robotic glossectomy for obstructive sleep apnea technique. Operative Techniques in Otolaryngology-Head and Neck Surgery. 2013;24(2):106-110. doi:10.1016/j.otot.2013.04.004.

17. Goyal N, Atmakuri M, Goldenberg D. Anatomy of the oropharynx: The robotic perspective. Operative Techniques in Otolaryngology-Head and Neck Surgery. 2013;24(2):70-73.

18. Friedman M, Salapatas AM, Bonzelaar LB. Updated Friedman Staging System for Obstructive Sleep Apnea. Adv Otorhinolaryngol. Basel. 2017;80:4148.

19. Vicini C, Montevecchi F, Tenti G. Transoral robotic surgery: Tongue base reduction and supraglottoplasty for obstructive sleep apnea. Operative Techniques in Otolaryngology. 2012;23:45-47.

20. Crawford JA, Montevecchi F, Vicini C, Magnuson JS. Transoral robotic sleep surgery: the obstructive sleep apnea-hypopnea syndrome. Otolaryngol Clin North Am. 2014;47(3):397-406. doi:10.1016/j. otc.2014.03.004.

21. Cammaroto G, Montevecchi F, D'Agostino G y cols. Tongue reduction for OSAHS: TORSs vs coblations, technologies vs techniques, apples vs oranges. Eur Arch Otorhinolaryngol. 2017;274(2):637-645. doi: 10.1007/s00405-016-4112-4.

22. Vicini C, Montevecchi F, Campanini A y cols. Clinical outcomes and complications associated with TORS for OSAHS: a benchmark for evaluating an emerging surgical technology in a targeted application for benign disease. ORL J Otorhinolaryngol Relat Spec. 2014;76(2):63-69. doi:10.1159/000360768.

23. Sethi RKV, Chen MM, Malloy KM. Complications of Transoral Robotic Surgery. Otolaryngol Clin North Am. 2020;S0030-6665(20)36641-X. doi:10.1016/j. otc.2020.07.017.

24. Friedman M, Hamilton C, Samuelson CG, y cols. Transoral robotic glossectomy for the treatment of obstructive sleep apnea-hypopnea syndrome. Otolaryngol Head Neck Surg. 2012;146(5):854-62. doi: 10.1177/0194599811434262.

25. Lin HS, Rowley JA, Folbe AJ, Yoo GH, Badr MS, Chen W. Transoral robotic surgery for treatment of obstructive sleep apnea: factors predicting surgical response. Laryngoscope. 2015;125(4):1013-1020. doi:10.1002/lary.24970.

26. Carney AS, Antic NA, Catcheside PG y cols. Sleep Apnea Multilevel Surgery (SAMS) trial protocol: a multicenter randomized clinical trial of upper airway surgery for patients with obstructive sleep apnea who have failed continuous positive airway pressure. Sleep. 2019;42(6):zsz056. doi:10.1093/sleep/zsz056.

27. Transoral Robotic Surgery: A Review of Clinical and Cost-Effectiveness. Ottawa (ON): Canadian Agency for Drugs and Technologies in Health; 2015.

28. Othman S, McKinnon BJ. Financial outcomes of transoral robotic surgery: A narrative review. Am J Otolaryngol. 2018;39(4):448-452. doi:10.1016/j. amjoto.2018.04.001.

29. Byrd JK, Paquin R. Cost Considerations for Robotic Surgery. Otolaryngol Clin North Am. 2020;S00306665(20)36643-3. doi:10.1016/j.otc.2020.07.019. 